Research Article

\title{
Magnitude of Intimate Partner Violence and Associated Factors among Pregnant Women in Ethiopia
}

\author{
Girmay Adhena $\mathbb{D D}^{1}{ }^{1}$ Lemessa Oljira, ${ }^{2}$ Yadeta Dessie $\mathbb{D}^{\circ},{ }^{2}$ and Hagos Degefa Hidru $\mathbb{D}^{3}$ \\ ${ }^{1}$ Reproductive Health Gambella Regional Health Bureau, Gambella, Ethiopia \\ ${ }^{2}$ School of Public Health, College of Health and Medical Science, Haramaya University, Harar, Ethiopia \\ ${ }^{3}$ Department of Public Health, College of Medical and Health Science, Adigrat University, Adigrat, Ethiopia \\ Correspondence should be addressed to Girmay Adhena; girmayrh@gmail.com
}

Received 27 November 2019; Revised 26 February 2020; Accepted 9 March 2020; Published 31 March 2020

Academic Editor: Jagdish Khubchandani

Copyright (c) 2020 Girmay Adhena et al. This is an open access article distributed under the Creative Commons Attribution License, which permits unrestricted use, distribution, and reproduction in any medium, provided the original work is properly cited.

\begin{abstract}
Background. Intimate partner violence during pregnancy is the most common and major public health problem and human rights issue worldwide and has a negative effect on the lives of both mother and fetus. Despite its prominence, this issue has received little attention in Ethiopia as well as many sub-Saharan African countries. This study assessed the magnitude of intimate partner violence and associated factors among pregnant women in Ofla District, Tigray, Ethiopia. Methods. A facility-based crosssectional study was conducted from March 1 to 30, 2019, among 543 pregnant women who visited antenatal care in the health facilities. A systematic random sampling technique was used to select study participants. Pretested, interviewer-administered data collection was done using a standardized World Health Organization multicountry questionnaire for women's health and domestic violence against women. Bivariable and multivariable logistic regression analyses were carried out to identify factors associated with violence. $p$ value was set at $p<0.05$. Results. The overall prevalence of intimate partner violence during the current pregnancy was 37.5\%: psychological (25.1\%), sexual (17.7\%), and physical violence (13.4\%). Violence was associated with unplanned pregnancy $((\mathrm{AOR}=4.56,95 \% \mathrm{CI}:(2,10.28))$, unmarried women $((\mathrm{AOR}=2.59,95 \% \mathrm{CI}:(1.18,5.73))$, having alcoholic partner $((\mathrm{AOR}=3.3,95 \% \mathrm{CI}:(2.1,5.16))$, spouse's multiple sexual partners status $((\mathrm{AOR}=5.1,95 \% \mathrm{CI}:(2.2,12))$, acceptance of violence by women $((\mathrm{AOR}=1.85,95 \% \mathrm{CI}:(1.1,3.16))$, low decision-making power of women $((\mathrm{AOR}=2.64,95 \% \mathrm{CI}:(1.6,4.3))$, and no interest in current pregnancy by partner $((\mathrm{AOR}=5.9,95 \% \mathrm{CI}:(2.36,14.9))$. Conclusions. More than one-third of pregnant women experienced intimate partner violence during a recent pregnancy. This is high and may lead to health consequences for both mothers and fetuses. Addressing gender inequitable norms, the culture of silence (support) to intimate partner violence in the community and women's reproductive health information through intervention measures are very important to minimize the problem.
\end{abstract}

\section{Background}

Intimate partner violence is defined as the intentional use of physical force or power threatened or actual against a woman or man within a relationship which either results in or has a high likelihood of resulting in injury, psychological harm, and death [1]. It is one of the most common forms of violence against women and major public health human rights issues that causes physical, sexual, and psychological harm including the act of physical aggression, sexual coercion, and psychological abuse by current or former intimate partners $[1,2]$. Intimate partner violence during pregnancy is the most devastating problem on both the mother and the fetus because of the acute morbidity and mortality associated with violence and its long-term impact on women and indirectly children's mental and physical health $[2,3]$.

Worldwide, 35\% of women have experienced physical and/or sexual violence by their intimate partner or nonintimate partner violence; $30 \%$ of these women have experienced physical and/or sexual violence by their intimate partner in their lifetime [4]. The lifetime prevalence of 
physical, sexual, or both physical and sexual violence ranges from $15 \%$ to $71 \%$ [5]. During pregnancy, the prevalence of intimate partner violence ranges from 1 to $28 \%[1,6]$. The millennium development goal was targeted to improve maternal health by reducing maternal mortality and universal access to reproductive health by 2015. Although substantial progress has been made globally, outcomes in the number of developing countries were limited [7]. The elimination of all forms of violence against women is one part of the recently adopted 2030 Agenda for Sustainable Development Goals (SDG) to achieve gender equality and to empower all women [4, 7].

Intimate partner violence is a global health issue that poses a greater risk for physical, sexual, and mental health problems and affects all the spheres of women's lives such as self-esteem, productivity, autonomy, capacity to care for themselves and their children, ability to participate in social activities, and even death [5]. Violence negatively affects women's reproductive health and may increase also the risk of acquiring human immune-deficiency virus (HIV) in some settings [6]. Pregnancy has been identified as a period of increased risk for exposure to violence, and the vulnerability of pregnant women to violence and to their consequences is an alarming public health issue in developing countries [8]. Intimate partner violence (IPV) during pregnancy is of concern as the possible consequences pose risk to both mother and the unborn child [2]. It has many adverse consequences including pregnancy-related complications (inadequate, labor complications, stillbirth, preterm delivery, induced abortion, and deaths), mental health problems and poor emotional well-being (emotional distress, depression, anxiety, low self-esteem, and poor attachment), cognitive and behavioral problems (increased aggression, antisocial behavior, lower social competence, and impaired cognitive functioning), behaviors presenting risks to health including alcohol and drug misuse and eating disorders, and physical injuries are some of them $[5,9-11]$. They are also at higher risk of being infected with sexually transmitted disease/HIV and harmful neonatal consequences such as low birth weight [1].

Although studies on violence against women in Africa are scarce, available data showed $36.6 \%$ of women in Africa experienced lifetime physical and/or sexual IPV among everpartnered women [1]. About 25\% in Ethiopia, 34\% in Zimbabwe, $61 \%$ in The Gambia, and 55.5\% in South Africa experienced physical/sexual IPV [12]. All over the world, sub-Saharan African (SSA) countries have recorded the highest levels of violence against women [2]. A systematic review in Africa showed that IPV during pregnancy ranged from $2.3 \%$ to $57.1 \%$ [13]. In many SSA countries including Ethiopia with high social acceptance of violence and poor socioeconomic status, the level of IPV during pregnancy estimated between $20 \%$ and $70 \%[1,11,14]$.

Even though Ethiopia has put in place appropriate and effective legal policies to promote the rights of women, violence against women continues to be a major challenge [7]. There is also a paucity of countrywide evidence about domestic violence against women in Ethiopia [15]. Women experienced $53.7 \%$ of IPV either physical or sexual or both within a year and $70.9 \%$ over their lifetime [1]. They are also characterized by low levels of education and lack of decisionmaking power which further makes them less assertive and more dependent on their male partners, thereby increasing their likelihood of experiencing violence during pregnancy [16]. Unplanned pregnancy and cultural attitudes towards violence by the community are also factors that appear to place in certain women at a somewhat greater risk to IPV $[15,16]$.

In Ethiopia, even though there is some improvement in decreasing violence against women, little attention is given for violence committed by intimate partners, especially for pregnant women. The majority of studies on IPV during pregnancy measure physical violence alone, although sexual and emotional violence during pregnancy is also considered as detrimental for women and their children's well-being. Moreover, studies on violence against women, particularly IPV during pregnancy, are limited in the study area. This study was therefore designed to assess the magnitude of intimate partner violence interims of physical, sexual, and psychological violence and associated factors among pregnant women during their current pregnancy.

\section{Methods}

2.1. Study Area and Period. The study was conducted in Ofla District which is one of the districts in Tigray Regional State, Northern Ethiopia. It is bounded in the south by Alamata, in the west by Amhara Region, in the north by Endamehoni, and in the east by Raya Azebo. Korem is the capital town of Ofla District, and it is located $619 \mathrm{~km}$ away from Adis Ababa, the capital city of Ethiopia. It has 24 kebeles ( 21 rural and 3 urban). The district also has a total population of 175,659 , of which 87,221 (49.65\%) were males and 88,438 (50.35\%) females. Regarding the health infrastructure, there is one general hospital, seven health centers, and 25 health posts that serve the population in the district. About 6413 pregnant women were followed during their antenatal clinic (ANC) in the public health facilities (seven health centers and one general hospital) in 2018 [17]. The study was conducted from March 1 to 30, 2019.

2.2. Study Design and Population. A facility-based crosssectional study was conducted. All pregnant women who were attending antenatal care (ANC) services in the public health facilities of Ofla District were the source population. Pregnant women who were receiving ANC in the public health facilities during the data collection period constituted the study population. Pregnant women with severe danger signs of pregnancy (vaginal bleeding and severe frontal headache) were excluded.

2.3. Sample Size Calculations and Sampling Procedures. Sample size was calculated for the first objective (magnitude) using single population formula $\left(n=z^{2} p(1-p) / d^{2}\right)$, where $z$ is the normal standard deviation set at 1.96 , with a confidence level specified at $95 \%$ and a tolerable margin of error (d) at 5\%, considering $10 \%$ nonresponse rate and prevalence 
of violence ( $p$ ) 25\% from a previous similar study in Gonder, Ethiopia [18]. The calculated sample size for this was 320 . The sample size was also calculated for the second objective (factors) by using the Epi-info version-7 with the assumption of $95 \%$ confidence interval, $80 \%$ power, $5 \%$ degree of precision, and $10 \%$ nonresponse rate. Since the sample size of the second objective (543) was higher than the first objective (320), the total sample in this study was 543 .

For the sampling procedure, first, all public health facilities (seven health centers and one general hospital) in Ofla District were considered. Pregnant women who attended ANC clinics for an average of six months in 2018 were used to estimate recruitment from each health facility. The average client flow per month and per day in the eight public health facilities of Ofla (Falla, Zata, Korem, Hashenge, Lat, Sesela, and Mymaido health centers and the Korem general hospital) was considered, and proportional allocation (PPS) was used. A systematic random sampling (SRS) technique was used to select study participants, and every third pregnant woman was chosen based on their visiting order until the sample size was met.

2.4. Data Collection Tool and Procedures. The standard WHO multicountry study questionnaire for assessing women's health and violence was used for data collection [5]. The questionnaire has three items for psychological violence, five items for physical violence, and three items for sexual violence, and the response to each item was either "Yes" or "No." Participants who respond "Yes" to one or more items of violence during recent pregnancy were considered as incident cases of intimate partner violence victimization. The questionnaire was translated into the local language (Tigrigna) and pretested on 5\% (27) of the total sample size was adapted and used. Data were collected by 10 midwives (who were all females, which creates an opportunity for disclosure of violence by the women). Two public health officers and the principal investigator supervised the data collection procedures. Data collectors and supervisors were trained for two days on interviewing techniques, the purpose of the study, the importance of privacy, the sensitivity of the issue, discipline and approach to the interviewees, and confidentiality of the respondents. The collected data were cross-checked on each day of activity for consistency and completeness, double-entry of data and cleaning of data using frequency, sorting, and listing to identify any missed value and outlier were made, and identified errors were cross-checked with the original questionnaire.

\subsection{Operational Definitions}

2.5.1. Intimate Partner Violence. Women who reported that they experienced any act of physical, sexual, or emotional (psychological) violence or any combination of the three by an intimate partner during current pregnancy [6].

2.5.2. Intimate Partner. Current husband, cohabited (live in the same house without formal marriage), or boyfriend [19].
2.5.3. Acceptance of $I P V$. Women were asked about their attitudes on IPV when they accepted violence committed by an intimate partner (argues with him, burns the food, goes without telling him, failing to complete housework, and refusal of men's request for sex). The response of "Yes" to one or more was considered as the woman accepts IPV positively, and the response of "No" to all was considered as the women do not accept IPV [20].

2.5.4. Physical Violence. Women who reported that they experienced one or more acts such as slapped, pushed, shoved, pulled, throw something that could hurt, choked, burning on purpose, hit abdomen with a fist or with something else and if a gun, knife, or any other weapon was used against woman by an intimate partner during current pregnancy [8].

2.5.5. Psychological Violence. Women who reported that they experienced one or more acts such as insult, humiliation, intimidate, or scared on purpose by an intimate partner during the current pregnancy [10].

2.5.6. Sexual Violence. Women who reported that they experienced one or more acts or threats such as forced into sexual intercourse when she did not want, had sexual intercourse when she did not want to because she was afraid of what partner might do, and forced to do something sexual that she found degrading or humiliating by an intimate partner during current pregnancy [18].

2.5.7. Decision-Making Power. The index for decisionmaking power composed of four questions. The women were asked, "who in her family usually has the final say on," how to use the money earned by her or her partner, healthcare for herself, major household purchases, and visits to her family or relatives. For each item, the response was with scores as 2 if a woman made a sole decision, 1 if she was involved with a partner or someone else, and 0 otherwise. The sum of the scores was made to represent an overall index of a woman's decision-making power. The total score on decision-making power was 8 . Hence, those women who scored four and above were categorized as having high decision-making power, whereas those scored less than four were categorized as women with low decision-making power [21].

2.6. Data Processing and Analysis. The data were first coded, entered, and cleaned using the Epi-data version 4.1 statistical software version and then exported into SPSS version 20 for analysis. Descriptive statistical analyses such as simple frequencies, measures of central tendency, and measures of variability were used to describe the characteristics of participants/partners. Then, information was presented using frequencies, summary measures, tables, and figures. The principal component analysis was used to produce wealth quintiles to show and reveal the socioeconomic status of the households. The bivariable analysis was carried out to see the 
association of each independent variable with the outcome variable (IPV). All variables with $p$ value $<0.25$ in the bivariable analysis were taken into the multivariable analysis model to control all possible confounders. The colinearity test was carried out to see the correlation between independent variables using the standard error. A Hosmer-Lemeshow and Omnibus tests were conducted to test model goodness of fit. The multivariable analysis was performed in the binary logistic regression up on controlling for the possible confounding factors. Odds ratios with 95\% CI were reported to show the strength and direction of the associations. Finally, variables with a $p$ value of less than 0.05 $(p<0.05)$ in the logistic regression were considered as statistically significant.

2.7. Ethical Approval. Ethical clearance was obtained from the Haramaya University College of Health and Medical Sciences Institutional Health Research Ethics Review Committee (IHRERC). Informed, voluntary, written, and signed consent was obtained from each head of the health facilities and each participant after clearly informing them about the purpose, risk, and benefit of the study. To assure the confidentiality of the study participants' information, the interview was conducted in a separate and calm room in each health facility.

\section{Results}

3.1. Descriptive Characteristics of Pregnant Women and Their Intimate Partners. A total of 538 pregnant women were interviewed, making a response rate of $99.08 \%$. The mean age of participants was $27.4( \pm 6.2)$ years. Near to half $(48.1 \%)$ of them were between the age of 25 and 34 years old. Three hundred and forty (63.2\%) of the participants were rural dwellers, 503 (93.5\%) were Orthodox Christian, and 531 (98.7\%) were Tigray in ethnicity. More than half of the pregnant women $(50.7 \%)$ had no formal education, onethird (33.4\%) lived into households with medium wealth quintile, and nearly half (48.9\%) were housewives. Regarding partners of pregnant women, about $244(45.4 \%)$ had no formal education, $28(5.2 \%)$ were smokers, and 58 $(10.8 \%)$ had another sexual partner. More than half $(54.1 \%)$ were alcohol users, and $73(13.6 \%)$ of partners did not desire the current pregnancy (Table 1).

\subsection{Reproductive and Other Characteristics of Pregnant} Women. Nearly nine out of ten $(88.5 \%)$ pregnant women were married, and $86(16 \%)$ women reported that the current pregnancy was unplanned. Regarding the capacity of decision making on household issues, one in three $(33.5 \%)$ participants had low discussion-making power (their intimate partner only decides on household issues), $18.6 \%$ did not discuss how to use money, $25.3 \%$ did not discuss health care for themselves, $23.6 \%$ did not discuss major household issues, and $25.7 \%$ did not discuss visiting their family. Three hundred and sixty (66.9\%) of participants reported acceptance of any form of violence by an intimate partner by justifying reasons of men's violent acts such as in the event of an argument, if she burns the food, goes without telling him, failing to complete housework, and refusal of men's request for sex (Table 1).

3.3. The Magnitude of Intimate Partner Violence. Among the 538 interviewed pregnant women, 202 (37.5\%; 95\% CI: $33.5 \%-41.6 \%)$ had experienced violence by their intimate partner during the recent pregnancy (Figure 1).

In this study, the prevalence of three forms of intimate partner violence during the current pregnancy was high. Psychological violence was the most common followed by sexual and physical violence. One hundred and thirty-five (25.1\%; 95\% CI: $21.2 \%, 29.3 \%)$ women reported psychological violence. Out of this number, insults and making her feel bad $(115(21.4 \%))$ was the most common followed by belittled or humiliated in front of others $(22(4.1 \%))$ and scared or intimidated on purpose $(40(7.4 \%))$ (Figure 2$)$.

Seventy-two ((13.4\%; 95\% CI: $(10.5 \%, 16.2 \%))$ pregnant women experienced physical violence. Out of this number, slapped, pushed, or hair-pulling was 51 (9.5\%), thrown something that could hurt her $(8(1.5 \%))$, hit in the abdomen with a fist or with something else that could hurt her (5 $(0.9 \%))$, choked or burnt on purpose (10 (1.9\%)), and threatened to use or actually used a gun, knife, or any other weapon against her $(12(2.2 \%))$ were reported. Ninety-five pregnant women $((17.7 \%$; $95 \% \mathrm{CI}$ : $(14.6 \%, 20.8 \%))$ reported sexual violence. Out of this number, $63(11.7 \%)$ reported to have been physically forced to have sexual intercourse, 63 $(11.7 \%)$ reported having unwanted sexual intercourse because of fear of partner, and 18 (3.3\%) reported having forced her to do something sexual that is degrading or humiliating during their current pregnancy (Figure 2).

This study also assessed the overlapping occurrence (more than one form) of violence against women. Women reported that psychological and physical violence $(8 \%)$, psychological and sexual violence (9.5\%), physical and sexual violence $(5.9 \%)$ and $4.8 \%$ of women experienced all forms of violence (psychological, physical and sexual). Among those pregnant women who experienced IPV, 171 $(84.65 \%)$ of them did not share the occurrence of violence to anyone else. In the majority of respondents, 469 (87.2\%) reported that no health care provider asked them about violence.

3.4. Factors Associated with Intimate Partner Violence during Pregnancy. In the bivariate analysis, women with no formal education, unemployed women, intimate partner with primary education, unmarried women, unplanned pregnancy, undesired pregnancy by partner, having alcohol drinking partner, having smoker partner, spouse's multiple sexual partners status, low discussion-making power of women, acceptance of IPV by women, and being in low- and medium-wealth quintile were the main significant factors associated with IPV. However, in the final model (multivariate analysis), the result showed that being unmarried, unplanned pregnancy, spouse's multiple sexual partners status, having alcohol drinker partner, undesired pregnancy by partner, acceptance of violence, and low decision-making 
TABLE 1: Socioeconomic, demographic, and other characteristics of pregnant women and their intimate partners, attending ANC at public health facilities in Ofla District, Tigray, Ethiopia, $2019(N=538)$.

\begin{tabular}{|c|c|c|c|}
\hline Variables & Category & Frequency & Percentages \\
\hline \multirow{3}{*}{ Age (years) } & $15-24$ & 193 & 35.9 \\
\hline & $25-34$ & 258 & 48 \\
\hline & $\geq 35$ & 87 & 16.1 \\
\hline \multirow{2}{*}{ Residence } & Rural & 340 & 63.2 \\
\hline & Urban & 198 & 36.8 \\
\hline \multirow{2}{*}{ Religion } & Orthodox & 503 & 93.5 \\
\hline & Muslim & 35 & 6.5 \\
\hline \multirow{2}{*}{ Ethnicity } & Tigray & 531 & 98.7 \\
\hline & Amhara & 7 & 1.3 \\
\hline \multirow{4}{*}{ Occupational status } & Housewife & 263 & 48.9 \\
\hline & Unemployed & 166 & 30.8 \\
\hline & Merchant & 57 & 10.6 \\
\hline & Government employee & 52 & 9.7 \\
\hline \multirow{3}{*}{ Educational status } & No formal education & 273 & 50.8 \\
\hline & Primary education & 133 & 24.7 \\
\hline & Secondary and above & 132 & 24.5 \\
\hline \multirow{3}{*}{ Educational status of partner } & No formal education & 244 & 45.4 \\
\hline & Primary education & 150 & 27.9 \\
\hline & Secondary and above & 144 & 26.7 \\
\hline \multirow{2}{*}{ Relationship status } & Married & 476 & 88.5 \\
\hline & Unmarried & 62 & 11.5 \\
\hline \multirow{2}{*}{ Plan of pregnancy } & Planned & 452 & 84 \\
\hline & Unplanned & 86 & 16 \\
\hline \multirow{2}{*}{ Decision-making power } & Low & 180 & 33.5 \\
\hline & High & 358 & 66.5 \\
\hline \multirow{2}{*}{ The desire for current pregnancy by partner } & Desired & 460 & 85.5 \\
\hline & Undesired & 78 & 14.5 \\
\hline \multirow{2}{*}{ Alcohol use (by partner) } & Yes & 291 & 54.1 \\
\hline & No & 247 & 45.9 \\
\hline \multirow{2}{*}{ Smoking status (by partner) } & Smokers & 28 & 5.2 \\
\hline & Nonsmokers & 510 & 94.8 \\
\hline \multirow{2}{*}{ Having another wife (by partner) } & Yes & 58 & 10.8 \\
\hline & No & 480 & 89.2 \\
\hline \multirow{2}{*}{ Acceptance of IPV } & Accept & 360 & 66.9 \\
\hline & Do not accept & 178 & 33.1 \\
\hline \multirow{3}{*}{ Wealth index } & Low & 179 & 33.3 \\
\hline & Medium & 180 & 33.4 \\
\hline & High & 179 & 33.3 \\
\hline
\end{tabular}

power of women remained the main significant factors associated with IPV (Table 2).

\section{Discussion}

Overall, the prevalence of IPV during the current pregnancy was $37.5 \%$ (95\% CI: 33.5\%-41.6\%). Unplanned pregnancy, being unmarried, acceptance of violence by women, discussion-making power of a woman in the household issues, having alcohol drinking partner, spouses multiple sexual partners status, and undesired current pregnancy by an intimate partner were significant factors associated with IPV during their current pregnancy.

This result (37.5\%) is in line with findings from Pakistan (35\%) [22] and Nigeria (34.3\%) [23] and a study in Ethiopia, Jimma $35.6 \%$ [19]. But this is lower as compared to the studies carried out in Zimbabwe (63.1\%) [24], Uganda (48\%) [25], South Africa (44\%) [26], Mozambique (44\%) [27], and Kenya (66.9\%) [28] and the studies conducted in Ethiopia, Abay Chamo (44.5\%) [11] and Gonder (58.7\%) [8]. The possible reason for the difference might be due to the difference in study designs. For example, a study conducted in Ethiopia, Abay Chamo [11], was a community-based study. However, this study was facility based which may miss those women who were not visiting the health facilities for antenatal care services. The difference might be due to the difference in duration. For example, studies in South Africa and Mozambique [26, 27] were focused on the lifetime prevalence of violence, but this study focused only on the current pregnancy. The difference could be also due to the difference in study subjects, as finding in Nigeria [23] was only rural dwellers and the study in Zimbabwe [24] was 


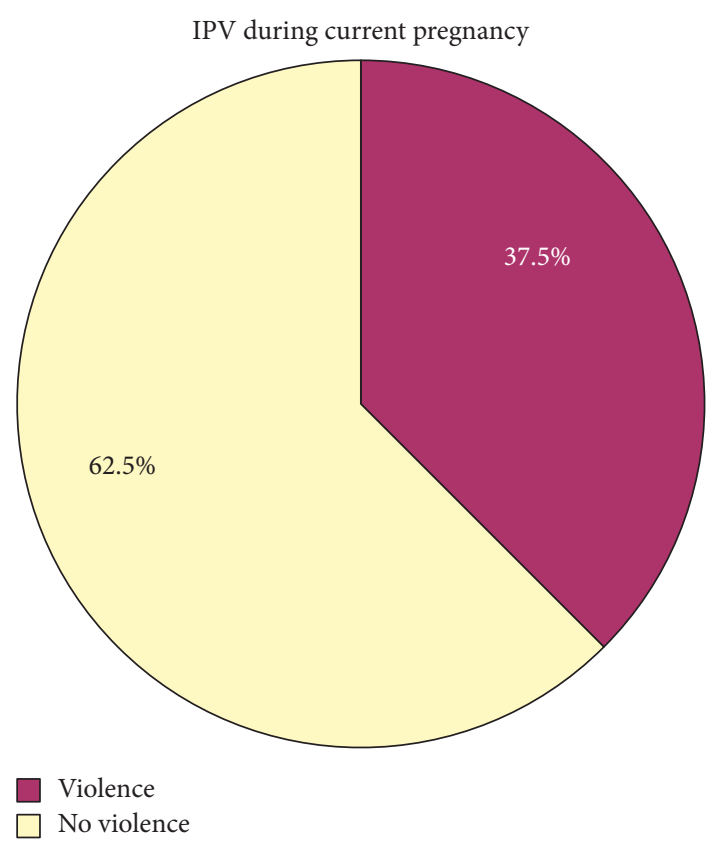

FIgURE 1: Magnitude of intimate partner violence among pregnant women attending antenatal care services at public health facilities in Ofla District, Tigray, Ethiopia, $2019(N=538)$.

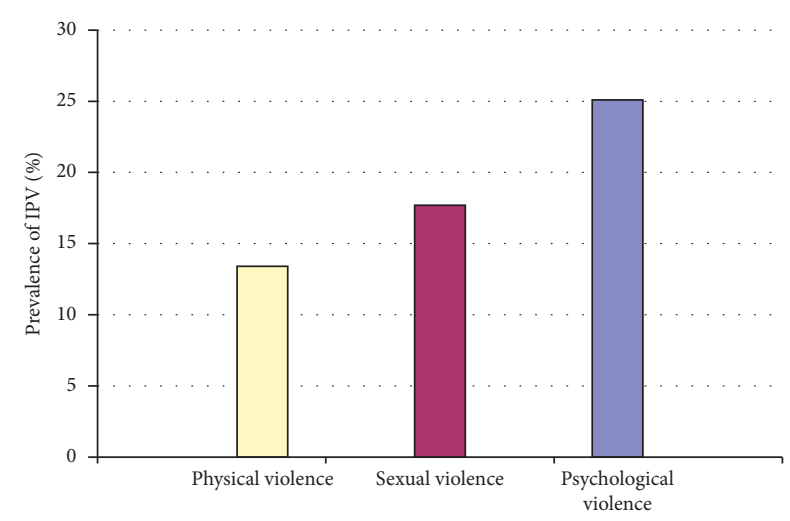

FIGURE 2: Magnitude of three forms of intimate partner violence among pregnant women attending antenatal care services at public health facilities in Ofla District, Tigray, Ethiopia, $2019(N=538)$.

focused only pregnant women with a lower socioeconomic status, this could result in the overestimation of the prevalence of the problem.

The prevalence of intimate partner violence in this study is more than two times compared to the findings in Nigeria (14.8\%) [29], Namibia (8\%) [3], South Africa (15\%) [30], and Brazil (15.5\%) [31] and a study in Yirgalem, Ethiopia, (12\%) [10]. It is also higher than the findings from Rwanda (29.2\%) [12] and Ethiopia (Shirendasilase (20.6\%) [9], Hulet Ejunesie (32.2\%) [32], and Gonder (25.4\%)) [18]. The possible explanation for the variation might be due to the difference in the accessibility of information on gender-based issues and reproductive health information and cultures of the study subjects. The other difference could also be that findings from Ethiopia (Shirendasilase [9] and Yirgalem [10]) measured only physical violence, but in this study, three types of violence (psychological, physical, and sexual violence) were included.

The association between respondents and their partner's sociodemographic and other-related characteristics and experiences of violence was assessed. The result showed that the likelihood of the occurrence of IPV was 2.6 times more likely among women who were unmarried compared to married women. This is supported by the findings from South Africa and the Demographic and Health Survey in sub-Saharan African countries [30,33]. The possible explanation could be that the married ones understand each other and are more likely to compromise on certain issues which brings less conflict in their homes. Another possible reason might be due to the sociocultural value in the communities, and some community gives high value to marriage.

The likelihood of intimate partner violence during the current pregnancy was 3.3 times more likely reported among IPV women whose husbands were alcohol users compared to those whose partners did not consume alcohol. These findings are supported by studies conducted in Kenya [28], in Arua, Uganda [25], and in Ethiopia (Shirendasilase [9], Gondar [8], and Huletejuensie [32]). This might be due to the fact that alcohol consumption directly affects consumers' cognitive and physical functions. This distortion in thinking might cause the users to behave aggressively in the relationship and increase the occurrence of violence.

This study also found a strong significant association of undesired pregnancy. Women whose partners did not desire the current pregnancy were about 5.9 times more likely to experience IPV compared to pregnant women whose partners desired the current pregnancy. This is consistent with the findings from Huletejuensie, Ethiopia [32]. The possible reason might be because women who experienced violence and undesired pregnancy mostly live in an environment with no good relationship. However, it should be noted that intimate partner violence may lead to unwanted pregnancy through coerced sex, thereby affecting a woman's ability to negotiate contraceptive use. Also, the abusive partner may refuse to use a condom or other family planning methods.

Pregnant women whose partners had other sexual partners were five times higher compared to women who reported that their partners did not have other sexual partners. This is consistent with the findings in Nigeria [23] and Uganda [25]. The possible reason might be due to the cultural values in which polygamy is impossible in the community. It could also be due to religious reasons for the community.

Women with low decision-making power who experience household issues were 2.6 times more likely to have experienced IPV than those who had a high capacity of decision-making in the household issues. This is consistent with a study conducted in Bangladesh [34]. The possible reason might be the culture of the communities that prefer women to be subordinated to men rather than making a sole decision. However, the finding is contrary to the findings of survey conducted in sub-Saharan African countries that women who had the capacity to take decisions were more 
TABLE 2: Factors independently associated with the experience of intimate partner violence during pregnancy in Ofla District, Tigray, Ethiopia, $2019(N=538)$.

\begin{tabular}{|c|c|c|c|c|}
\hline \multirow{2}{*}{ Variables } & \multicolumn{2}{|c|}{ Violence (IPV) } & \multirow{2}{*}{ COR (95\% CI) } & \multirow{2}{*}{ AOR (95\% CI) } \\
\hline & Yes & No & & \\
\hline \multicolumn{5}{|c|}{ Women's educational status } \\
\hline No formal education & $115(42.1)$ & $158(57.9)$ & $1.5(0.97,2.33)$ & $0.794(0.34,1.857)$ \\
\hline Primary (grade $1-8$ ) & $44(33.1)$ & $89(66.9)$ & $1.02(0.61,1.71)$ & $0.77(0.35,1.73)$ \\
\hline High school and above & $43(32.6)$ & $89(67.4)$ & 1 & 1 \\
\hline \multicolumn{5}{|l|}{ Women's occupation } \\
\hline Government employee & $16(30.8)$ & $36(69.2)$ & 1 & 1 \\
\hline Merchant & $14(24.6)$ & $43(75.4)$ & $0.73(0.32,1.7)$ & $0.719(0.22,2.35)$ \\
\hline Housewife & $97(36.9)$ & $166(63.1)$ & $1.32(0.69,2.49)$ & $1.42(0.502,3.99)$ \\
\hline Unemployed & $75(45.2)$ & $91(54.8)$ & $1.85(0.96,3.6)$ & $1.17(0.425,3.205)$ \\
\hline \multicolumn{5}{|c|}{ Educational status of partner } \\
\hline No formal education & $103(42.2)$ & $141(57.8)$ & $1.22(0.8,1.86)$ & $0.929(0.49,1.74)$ \\
\hline Primary (grade $1-8$ ) & $45(30)$ & $105(70)$ & $0.71(0.44,1.16)$ & $0.57(0.29,1.13)$ \\
\hline High school and above & $54(37.5)$ & $90(62.5)$ & 1 & 1 \\
\hline \multicolumn{5}{|l|}{ Relationship status } \\
\hline Married & $161(33.8)$ & $315(66.2)$ & 1 & 1 \\
\hline Unmarried & $41(66.1)$ & $21(33.9)$ & $3.8(2.04-4.67)$ & $2.597(1.178,5.73)^{*}$ \\
\hline \multicolumn{5}{|l|}{ Plan of pregnancy } \\
\hline Unplanned & $75(87.2)$ & $11(12.8)$ & $17.5(8.97-33.9)$ & $4.56(2.02,10.3)^{* *}$ \\
\hline Planned & $127(28.1)$ & $325(71.9)$ & 1 & 1 \\
\hline \multicolumn{5}{|c|}{ Desiredness of current pregnancy by partner } \\
\hline Undesired & $70(89.7)$ & $8(10.3)$ & $21.7(10.2,46.4)$ & $5.95(2.36,14.99)^{* *}$ \\
\hline Desired & $132(28.7)$ & $328(71.3)$ & 1 & 1 \\
\hline \multicolumn{5}{|l|}{ Partner drinks alcohol } \\
\hline Yes & $155(53.3)$ & $136(46.7)$ & $4.85(3.28,7.18)$ & $3.27(2.07,5.16)^{*}$ \\
\hline No & $47(19)$ & $200(81)$ & 1 & 1 \\
\hline \multicolumn{5}{|c|}{ Partner smoking cigarette status } \\
\hline Smoker & $23(82.1)$ & $5(17.9)$ & $8.5(3.18,22.76)$ & $2.24(0.56,9.02)$ \\
\hline Nonsmoker & $179(35.1)$ & $331(64.9)$ & 1 & 1 \\
\hline \multicolumn{5}{|l|}{ Partner has another wife } \\
\hline Yes & $48(82.8)$ & $10(17.2)$ & $10.2(5.01,20.6)$ & $5.14(2.2,12)^{*}$ \\
\hline No & $154(32.1)$ & $326(67.9)$ & 1 & 1 \\
\hline \multicolumn{5}{|c|}{ Decision-making power (woman) } \\
\hline Low decision-making & $107(59.4)$ & $73(40.6)$ & $4.06(2.78,5.93)$ & $2.64(1.63,4.28)^{* *}$ \\
\hline High decision-making & $95(26.5)$ & $263(73.5)$ & 1 & 1 \\
\hline \multicolumn{5}{|c|}{ Acceptance of IPV by pregnant } \\
\hline Accept & $164(45.6)$ & $196(54.4)$ & $3.08(2.04,4.67)$ & $1.85(1.088,3.157)^{*}$ \\
\hline Do not accept & $38(21.4)$ & $140(78.6)$ & 1 & 1 \\
\hline \multicolumn{5}{|c|}{ Economic status (wealth index) } \\
\hline Low & $49(27.4)$ & $130(72.6)$ & $0.35(0.22,0.54)$ & $0.635(0.362,1.13)$ \\
\hline Medium & $60(33.3)$ & $120(66.7)$ & $0.46(0.3,0.71)$ & $0.611(0.357,1.05)$ \\
\hline High & $93(51.9)$ & $86(48.1)$ & 1 & 1 \\
\hline
\end{tabular}

${ }^{*}=p$ value $<0.05 ;{ }^{* *}=p$ value $<0.01 ; \mathrm{CI}=$ confidence interval; $\mathrm{COR}=$ crude odds ratio; $\mathrm{AOR}=$ adjusted odds ratio.

likely to experience IPV compared to those who did not have decision-making capacity in the household issues [33]. The possible explanation for this might be women who are more empowered are able to fight for their rights and will not accept men to fully dictate to them, which could result in violence.

Women who accept any form of violence by intimate partners by justifying reasons were 1.9 times more likely to have experienced violence compared to those who did not accept violence by justifying reasons. This is consistent with the findings in Nepal [35] and the Demographic and Health
Survey conducted from six African countries [33]. The possible reason might be due to the socioculture of the community norms that privilege gives higher status to men and lower status to women and considered IPV as normal, and this may increase violence.

Those women with unplanned pregnancy experienced intimate partner violence around 4.6 times higher compared to women with planned pregnancy in this study. This is consistent with the findings in Ethiopia (Gonder [18] and Yirgalem [10]). This may be due to the fear of taking the responsibility to care for both the mother and the newly 
coming child, or this unplanned pregnancy might be also due to the result of sexual violence.

\section{Limitations}

There might be under-reporting due to the sensitive nature of intimate partner violence and cultural barriers to disclosing partners' issues to third parties. Data were also collected from service users at clinics. This means that nonusers of antenatal services, who might be the most vulnerable to intimate partner violence, were not included in the sample study.

\section{Conclusion}

Overall, more than one-third (37.5\%) of the pregnant women reported intimate partner violence during the current pregnancy. From those, psychological violence (25.1\%), sexual violence $(17.7 \%)$, and physical violence (13.4\%) were experienced. This is high, which may lead to health consequences both on the mothers and on their fetuses. Unplanned pregnancy, unmarried women, having an alcoholic user partner, spouse's multiple sexual partners, acceptance of IPV by women, low decision-making power of women, and the desire for the current pregnancy by a partner were found to be the main factors affecting intimate partner violence during the current pregnancy. The Ofla District Health Office in collaboration with the southern Tigray zone and Tigray regional health bureau should provide training on IPV for health care providers to screen and give holistic care to violence victims, strengthen the provision of reproductive health programs at the community level, and integrate the justice and health sectors to ensure a comprehensive service response through strengthened laws, well-organized legal systems, and enforcement mechanisms to decrease violence against women. Moreover, local councils, women's groups, mother's unions, leadership committees, health extension workers, and NGOs should also work on increasing awareness of the community to break the culture of silence on IPV. Further longitudinal research is recommended to assess the attitudes and perceptions of pregnant women towards IPV.

\section{Abbreviations}

ANC: Antenatal care

DHS: Demographic and health survey

IPV: Intimate partner violence

NGO: Nongovernmental organization

SPSS: Statistical package for social sciences

SSA: Sub-Saharan Africa

WHO: World Health Organization.

\section{Data Availability}

The datasets used and/or analyzed during the current study are available from the principal author upon reasonable request.

\section{Ethical Approval}

Before the data collection process, ethical clearance was secured from the Haramaya University Institutional Health Research Ethics Review Committee (IHRERC). The official letter was written from Haramaya University to the Ofla District Health Office and to each health facility.

\section{Consent}

Informed written consent was obtained from each participant after explaining the purpose and benefits of the study. Respondents were informed that participating in this study is up to the willingness of them.

\section{Conflicts of Interest}

The authors declare that they have no conflicts of interest.

\section{Authors' Contributions}

GA was involved in conception and design of the study, acquisition of data, analysis of data, interpretation of data, revising the paper, and writing of the manuscript). LO, YD, and $\mathrm{HDH}$ were involved in advising during proposal development, data analysis, and revising the paper. All authors read and approved the final manuscript.

\section{Acknowledgments}

The authors are grateful to the Haramaya University College of Health and Medical Sciences, Ofla/Korem District Health Office, for their cooperation and support, data collectors and supervisors for their responsible data collection and support, and all the study participants, particularly those pregnant women who disclosed or let us know their painful violence experiences. All costs of data collection and analysis were covered by the authors.

\section{References}

[1] WHO, Global and Regional Estimates of Violence against Women: Prevalence and Health Effects of Intimate Partner Violence and Non-partner Sexual Violence, Reproductive Health and Research, Geneva, Switzerland, 2013, http://apps. who.int/iris/bitstream/10665/85240/1/9789241548595_eng.

[2] K. M. Devries, J. Y. T. Mak, C. García-Moreno et al., "The global prevalence of intimate partner violence against women," Science, vol. 340, no. 6140, pp. 1527-1528, 2013.

[3] L. T. Bikines, R. Mash, and K. Joyner, "Prevalence of intimate partner violence and associated factors amongst women attending antenatal care at Outapi clinic, Namibia," African Journal of Primary Health Care \& Family Medicine, vol. 9, pp. 1-6, 2017.

[4] WHO, United Nations Development Programme; United Nations Office on Drugs and Crime. Global Status Report on Violence Prevention, http://www.who.int/violence_injury_ prevention/violence/status_report/2014/en/, WHO, Geneva, Switzerland, 2014, http://www.who.int/violence_injury_ prevention/violence/status_report/2014/en/.

[5] C. García-Moreno, H. Jansen, M. Ellsberg, L. Heise, and C. H. Watts, WHO Multi-Country Study on Women's Health and Domestic Violence against Women: Initial Results on 
Prevalence, Health Outcomes and Women's Responses, WHO, Geneva, Switzerland, 2005.

[6] WHO, "Understanding and addressing violence against women," Intimate Partner Violence, RHR, Geneva, Switzerland, 2012.

[7] EDHS, "Central statistical agency," Ethiopian Demographic Health Survey Addis Ababa, Ethiopia and Rockville, CSA and ICF, Rockville, MD, USA, 2016.

[8] E. Fekadu, G. Yigzaw, K. A. Gelaye et al., "Prevalence of domestic violence and associated factors among pregnant women attending antenatal care service at University of Gondar Referral Hospital, Northwest Ethiopia," Biomed Central Women's Health, vol. 18, pp. 1-8, 2018.

[9] B. H. Gebrezgi, M. B. Badi, E. A. Cherkose, and N. B. Weldehaweria, "Factors associated with intimate partner physical violence among women attending antenatal care in Shire Endaselassie town, Tigray, northern Ethiopia: a crosssectional study," Reproductive Health, vol. 14, no. 1, p. 76, 2017.

[10] Y. K. Zemenu and W. M. Alemu, "Physical violence and associated factors during pregnancy in Yirgalem town, South Ethiopia," Current Pediatrics Research, vol. 20, pp. 37-42, 2016.

[11] B. A. Abebe, W. BitiyaAdmassu, and T. D. Tizta, "Determinants of intimate partner violence during pregnancy among married women in Abay Chomen district, Western Ethiopia: a community-based cross-sectional study," BMC Women's Health, vol. 16, no. 1, 2016.

[12] A. A. Rurangirwa, I. Mogren, J. Ntaganira, and G. Krantz, "Intimate partner violence among pregnant women in Rwanda, its associated risk factors and relationship to ANC services attendance: a population-based study," BMJ Open, vol. 7, no. 2, Article ID e013155, 2017.

[13] S. Shamu, N. Abrahams, M. Temmerman, A. Musekiwa, and C. Zarowsky, "A systematic review of African studies on intimate partner violence against pregnant women: prevalence and risk factors," PLoS One, vol. 6, no. 3, Article ID e17591, 2011.

[14] A. Semahegn and B. Mengistie, "Domestic violence against women and associated factors in Ethiopia; a systematic review," Reproductive Health, vol. 12, p. 78, 2015.

[15] CSA, Ethiopia and ORC Macro, Ethiopian Demographic Health Survey Maryland: Ethiopia and Calverton, CSA, Addis Ababa, Ethiopia, 2012.

[16] S. G. Abeya, M. F. Afework, and A. W. Yalew, "Intimate partner violence against women in west Ethiopia. On attitudes, woman's response, and suggested measures as perceived by community members," Reproductive Health, vol. 9, p. 14, 2012.

[17] Ofla District Health Office Annual Report, 2018.

[18] B. B. Berhanu, A. D. Berihun, T. T. Bewket, and Z. Z. Abraham, "Domestic violence among pregnant mothers in Northwest Ethiopia: prevalence and associated factors," Advances in Public Health, vol. 2017, Article ID 6506231, 6 pages, 2017.

[19] B. T. Gashaw, B. Schei, and H. J. Magnus, "Social-ecological factors and intimate partner violence in pregnancy," PLoS One, vol. 13, no. 3, Article ID e0194681, 2018.

[20] C. D. Trott, J. J. Harman, and M. R. Kaufman, "Women's attitudes toward intimate partner violence in Ethiopia: the role of social norms in the interview context," Violence Against Women, vol. 23, no. 8, pp. 1016-1036, 2017.

[21] A. Eshetu and S. Yohannes, "Disparities in intimate partner violence among currently married women from food secure and insecure urban households in South Ethiopia: a community based comparative cross-sectional study," BioMed Research International, vol. 2018, Article ID 4738527, 12 pages, 2018.

[22] N. Ali, F. Ali, A. Khuwaja, and K. Nanji, "Factors associated with intimate partner violence against women in a megacity of South-Asia: a multi-center cross-sectional study," Hong Kong Medicine Journal, vol. 20, pp. 297-303, 2014.

[23] A. Ashimi and T. Amole, "Prevalence and predictors for domestic violence among pregnant women in a rural community Northwest, Nigeria," Nigerian Medical Journal, vol. 56, no. 2, pp. 118-121, 2015.

[24] S. Shamu, N. Abrahams, C. Zarowsky, T. Shefer, and M. Temmerman, "Intimate partner violence during pregnancy in Zimbabwe: a cross-sectional study of prevalence, predictors and associations with HIV," Tropical Medicine \& International Health, vol. 18, no. 6, pp. 696-711, 2013.

[25] A. Namugamba and R. Mangwi, "Prevalence and factors associated with domestic violence during pregnancy in Arua district, Uganda, 2015," Iproceedings, vol. 4, no. 1, 2018.

[26] M. Malan, M. F. Spedding, and K. Sorsdahl, "The prevalence and predictors of intimate partner violence among pregnant women attending a midwife and obstetrics unit in the Western Cape," Global Mental Health, vol. 5, pp. 1-13, 2018.

[27] E. Chaquisse, S. Fraga, P. Meireles et al., "Sexual and physical intimate partner violence among women using antenatal care in Nampula, Mozambique," Journal of Public Health in Africa, vol. 9, p. 744, 2018.

[28] O. O. Isaac, K. N. Margaret, and E. A. Harryson, "Intimate partner violence in pregnancy among antenatal attendees at health facilities in West Pokot County, Kenya," Pan African Medical Journal, vol. 28, p. 229, 2017.

[29] S. Anzaku, A. R. J. Shuaibu, M. Dankyau, and G. Chima, "Intimate partner violence and associated factors in an obstetric population in Jos, North-central Nigeria," Sahel Medical Journal, vol. 20, no. 2, pp. 49-54, 2017.

[30] S. Field, M. Onah, T. Heyningen, and S. Honikman, "Domestic and intimate partner violence among pregnant women in a low resource setting in South Africa," BMC Women's Health, vol. 18, pp. 1-13, 2018.

[31] D. P. Rodrigues, F. A. Gomes-Sponholz, J. Stefanelo, A. M. S. Nakano, and J. C. d. S. Monteiro, "Intimate partner violence against pregnant women: study about the repercussions on the obstetric and neonatal results," Revista da Escola de Enfermagem da USP, vol. 48, no. 2, pp. 206-212, 2014.

[32] Y. Tenaw, G. Tesfaye, E. Gudina, and M. Habtamu, "Magnitude of Domestic Violence and Associated Factors Among Pregnant Women in Hulet Ejju Enessie District, Northwest Ethiopia," Advances in Public Health, vol. 2014, Article ID 484897, 8 pages, 2014.

[33] B. O. Ahinkorah, K. S. Dickson, and A.-A. Seidu, "Women's decision-making capacity and intimate partner violence among women in sub-Saharan Africa," $B M C$, vol. 5, pp. 1-10, 2018.

[34] M. S. Alam, M. I. Tareque, E. D. Peet, M. M. Rahman, and T. Mahmud, "Female participation in household decision making and the justification of wife beating in Bangladesh," Journal of Interpersonal Violence, pp. 1-20, 2018.

[35] K. Yoshikawa, T. M. Shakya, K. C. Poudel, and M. Jimba, "Acceptance of wife beating and its association with physical violence towards women in Nepal: a cross-sectional study using couple's data," PLoS One, vol. 9, no. 4, Article ID e95829, 2014 